NOTICE: this is the author's version of a work that was accepted for publication in Journal of Fluids and Structures. Changes resulting from the publishing process, such as peer review, editing, corrections, structural formatting, and other quality control mechanisms may not be reflected in this document. Changes may have been made to this work since it was submitted for publication. A definitive version was subsequently published in Journal of Fluids and Structures, Vol. 48 (2014). DOI: 10.1016/j.jfluidstructs.2014.03.012 


\title{
Stability of a flexible insert in one wall of an inviscid channel flow
}

\author{
Meagan A. Burke ${ }^{\mathrm{a}, *}$, Anthony D. Lucey ${ }^{\mathrm{a}}$, Richard M. Howell ${ }^{\mathrm{a}}$, Novak S. J. Elliott ${ }^{\mathrm{a}}$ \\ ${ }^{a}$ Fluid Dynamics Research Group, Department of Mechanical Engineering, Curtin University, P.O. Box U1987, Perth 6845, \\ Australia
}

\begin{abstract}
A hybrid of computational and theoretical methods is extended and used to investigate the instabilities of a flexible surface inserted into one wall of an otherwise rigid channel conveying an inviscid flow. The computational aspects of the modelling combine finite-difference and boundary-element methods for structural and fluid elements respectively. The resulting equations are coupled in state-space form to yield an eigenvalue problem for the fluid-structure system. In tandem, the governing equations are solved to yield an analytical solution applicable to inserts of infinite length as an approximation for modes of deformation that are very much shorter than the overall length of the insert. A comprehensive investigation of different types of inserts - elastic plate, damped flexible plate, tensioned membrane and spring-backed flexible plate - is conducted and the effect of the proximity of the upper channel wall on stability characteristics is quantified. Results show that the presence of the upper-channel wall does not significantly modify the solution morphology that characterises the corresponding open-flow configuration, i.e. in the absence of the rigid upper channel wall. However, decreasing the channel height is shown to have a very significant effect on instability-onset flow speeds and flutter frequencies, both of which are reduced. The channel height above which channel-confinement effects are negligible is shown to be of the order of the wavelength of the critical mode at instability onset. For spring-backed flexible plates the wavelength of the critical mode is much shorter than the insert length and we show very good agreement between the predictions of the analytical and the state-space solutions developed in this paper. The small discrepancies that do exist are shown to be caused by an amplitude modulation of the critical mode on an insert of finite length that is unaccounted for in the travelling-wave assumption of the analytical model. Overall, the key contribution of this paper is the quantification of the stability bounds of a fundamental fluid-structure interaction (FSI) system which has hitherto remained largely unexplored.
\end{abstract}

Keywords:

Fluid-structure interaction; Boundary-element method; Finite-difference method; Eigen-analysis; State-space solution; Divergence; Flutter.

\section{Introduction}

This paper describes the modelling and prediction of the flow-induced instabilities experienced by a finite flexible wall inserted in one side of an otherwise rigid-walled two-dimensional channel conveying a fluid flow; the system studied is depicted in Fig. 1. We assume a potential flow thereby making the results relevant to flows with very high (effectively infinite) Reynolds number that would be typically encountered in engineered systems as opposed to the biomechanical applications that usually motivate study of the system in Fig. 1. However, there remain some biomechanical applications for which the present findings may be useful; these are discussed below. The system studied is also related to that of fluid-conveying pipes for which there exists a rich literature motivated by many industrial applications such as those found in process engineering.

\footnotetext{
${ }^{*}$ Corresponding author. Tel: +61892667047

Email address: m.burke@postgrad.curtin.edu.au (Meagan A. Burke)
} 
Accordingly, the present system may be considered to sit at the intersection of a number of well-studied fundamental problems that we briefly review in this introduction. The overarching purpose of this paper is to characterise the fundamental fluid-structure interaction (FSI) of a system that has hitherto received very little attention when the flow, in practical terms, may be considered inviscid.

In the absence of the upper channel wall in Fig. 1, the system is immediately recognised as the classical problem of panel flutter first studied for aeronautical applications (Bisplinghoff et al., 1955; Dugundji et al., 1963). In marine environments, studies of compliant walls or flexible panels were motivated by the experiments of Kramer (1960) that suggested wall compliance could reduce skin-friction by delaying boundary-layer laminar-to-turbulent transition; for examples, see Carpenter and Garrad (1985) and Carpenter et al. (2000). Other applications, such as unstable vibrations of steel (flexible) plates of spillways in hydro-power plants and hull panels of modern high-speed ships have continued to motivate studies of the fundamental system. Therefore, a variety of methods have been developed for this problem. Early studies, such as those of Weaver and Unny (1970) and Kornecki et al. (1976) used a Galerkin approach to predict instabilities, whereas Carpenter and Garrad (1986) used a travelling-wave approach, and numerical simulations were performed by Lucey and Carpenter (1992). Recently, Pitman and Lucey (2009) presented a versatile method by which system eigenvalues can be directly extracted even for structurally inhomogeneous systems. The broad consensus of these linear studies for incompressible, inviscid flow is that a flexible panel or compliant wall first loses its stability to divergence, while at higher speeds divergence recovery may occur, but at sufficiently high flow speeds a modal-coalescence type of flutter dominates the system response.

The aforementioned analytical solution methods are based upon a normal-mode decomposition of system disturbances. The Galerkin approach constructs a system solution as the sum of a set of discrete orthogonal functions usually chosen as the in vacuo structural modes because they each automatically satisfy the boundary conditions of the structural side of the system. For walls of infinite length, the travelling-wave assumption invokes a continuous spectrum of locally defined normal modes to characterise system disturbances. The ensuing solutions then predict instability in the limit of infinite time; i.e. as a boundary-value problem, in the form of the most unstable system eigenmode. However, recent work shows that this is not necessarily the route to large-amplitude deformations. Schmid and de Langre (2003) and Coppola and de Luca (2010) demonstrated theoretically that very significant transient growth of perturbations can occur through the non-normality of the system equations. Theoretical predictions of this type of phenomenon were confirmed in the experimental studies of Hémon et al. (2006) and Schwartz et al. (2009). The theoretical framework established in these non-modal analyses maximises the time-evolution of an energy norm for the fluid-structure system as an envelope over all potential initial states. This captures the growth of disturbances that can bypass conventional linear-instability mechanisms to reach finite amplitudes which may occur for system control parameters (e.g. the flow speed) below the critical values based on a modal analysis. This line of investigation is not pursued in the present paper although the methods we deploy are amenable to this type of study. Thus, for example, Tsigklifis and Lucey (2013) have recently extended the modelling of Pitman and Lucey (2009), upon which the present analysis is based, to analyse non-modal transient growth in boundary-layer flow over a compliant panel.

With a flexible insert comprising the wall of a channel, the present work bears similarity with studies of fluid conveying flexible pipes. Pipe-buckling (divergence) and flutter have been predicted at sufficiently high flow speeds, for example, by de Langre and Ouvrard (1999) and Doaré and de Langre (2002), in an infinitely long pipe comprising parallel flexible surfaces in the undeformed state. In many of these studies, a one-dimensional (or plug) flow is assumed in which the flow follows the curve of the deformed channel (or pipe) via the Païdoussis equation (Païdoussis, 1998, 2003). Of closer similarity to the present work that features flow curvature varying across the channel (the $y$-direction in Fig. 1) is the study of Weaver and Païdoussis (1977) that modelled two-dimensional potential flow in an infinitely long flexible channel using a travelling-wave assumption of disturbances in the streamwise direction and a Galerkin approach for a finite section of the channel. They found that: (i) when the walls display sinuous behaviour, divergence-onset flow speed increases as the channel height decreases; and (ii) when the walls display varicose behaviour, divergence-onset flow speed decreases as the channel height decreases. The first can be explained by the decrease in destabilising centrifugal force for a reduced mass of fluid traversing the curved path of a channel deformation. The second can be understood as arising from an increase in the gradient of flow curvature 
across the channel forced by a reduction in channel height; similar effects are demonstrated in the present work and will be discussed later in this paper.

Experimental work that complements the aforementioned pipe- and channel-flow modelling includes that of Dodds and Runyan (1965) who found a critical flow velocity for static divergence for a high-velocity fluid through a simply supported pipe and Weaver and Païdoussis (1977) who observed a flapping instability in a flattened tube and found that the critical flow speed at which flapping occurred was reduced with the gap between tube surfaces. However, most experimental studies - for examples see Conrad (1969), Gavriely et al. (1989), Bertram et al. (1990), Bertram and Castles (1999) and Bertram and Elliott (2003) - have tended to focus on large-amplitude deformations and the collapse of flexible channels in the context of biomechanical applications such as blood-flow in the smaller vessels. Equally then, there is a large body of theoretical and computational modelling on viscous flow in channels and pipes. These effectively address the low-Reynoldsnumber range - unsteady laminar-flow - of fluid-structure interactions within the canonical system. The system is shown to support a range of instabilities that include divergence, travelling-wave flutter, TollmienSchlichting waves and non-linear self-excited oscillations. Linear deformations were considered by Davies and Carpenter (1997) for an infinitely long flexible channel showing, inter alia, that different instabilities, for example Tollmien-Schlichting waves and travelling-wave flutter, can interact with each other. Huang (2001) modelled small-amplitude deformations of a finite tensioned membrane, found the eigenmodes of divergence and flutter and considered the effect of the membrane properties and up- and down-stream channel length on the system while Jensen and Heil (2003) developed predictions for the frequency and growth rate of instabilities. Models for large-amplitude deformations of a finite membrane in a channel have been developed by Luo and Pedley $(1996,2000)$ to simulate sustained self-excited oscillations of the membrane. Recent reviews of the rich body of work in this field have been presented by Grotberg and Jensen (2004) and Heil and Hazel (2011). The restriction to small-amplitude wall disturbances and inviscid flow in the present work render its biomechanical applications to lie in the approximate modelling of fluid-structure interactions in human upper-airway dynamics and in only the largest blood vessels.

The overall goal of this paper is to present a comprehensive picture of the stability characteristics of the system in Fig. 1 focusing on its parametric dependence upon channel height for a range of flexible inserts that include simple flexible plates, tensioned membranes and spring-backed flexible plates (compliant coatings). To do this, an extension of the method of Pitman and Lucey (2009) is developed whereby system eigenvalues are extracted using a hybrid of theoretical and computational methods. The present modelling is validated by comparison of its predictions at asymptotic limits with those of other fully analytical solutions that can be found for simplified forms of the configuration shown in Fig. 1. Overall, the present paper shows that while channel-confinement effects do not alter the phenomenology of the system behaviour well-described for open flows, they do exercise a significant effect on stability bounds and critical flutter frequencies. The quantitative characterisation of these effects - relative to corresponding open-flow systems - contributes a definitive set of non-dimensional stability data for channel flows past a flexible insert at the limit of infinite Reynolds number. Accordingly, this data-set can serve as baseline for future studies addressing turbulent channel flow past a flexible insert at very high Reynolds numbers wherein viscous effects are largely confined to a thin near-wall layer and the FSI dynamics are dominated by the potential-flow core.

\section{Method}

\subsection{Governing equations}

The motion of the flexible insert is described by the extended one-dimensional beam equation,

$$
\rho_{\mathrm{m}} h \frac{\partial^{2} \eta}{\partial t^{2}}+d \frac{\partial \eta}{\partial t}+B \frac{\partial^{4} \eta}{\partial x^{4}}+K \eta-T \frac{\partial^{2} \eta}{\partial x^{2}}=-\triangle p(x, y, t),
$$

where $\eta(x, t), \rho_{\mathrm{m}}, h, d$ and $B$ are respectively the plate's vertical displacement, density, thickness, damping and flexural rigidity, $K$ is the stiffness of the uniformly distributed spring foundation, $T$ is a uniform tension and $\Delta p$ is the unsteady pressure perturbation. Hinged boundary conditions are applied to the ends of the flexible insert. 
Assuming an irrotational and incompressible flow allows the introduction of a velocity perturbation $\phi(x, y, t)$ which satisfies Laplace's equation,

$$
\nabla^{2} \phi=0 .
$$

The fluid pressure is found from the unsteady Bernoulli equation,

$$
\Delta p=-\rho_{\mathrm{f}} U \frac{\partial \phi}{\partial x}-\rho_{\mathrm{f}} \frac{\partial \phi}{\partial t},
$$

where $\rho_{\mathrm{f}}$ and $U$ are respectively the fluid density and flow speed. The linearised (for small interfacial displacement) fluid-solid boundary conditions are,

$$
\begin{aligned}
\frac{\partial \phi}{\partial y}=0 \quad \text { at } & y=H, \text { and } y=0 \text { for } x<0 \text { and } x>L \\
\frac{\partial \phi}{\partial y}=\frac{\partial \eta}{\partial t}+U \frac{\partial \eta}{\partial x} \quad \text { at } & y=0,0 \leq x \leq L,
\end{aligned}
$$

where $H$ is the channel height.

We remark that the formulation described above assumes that the pressure of the mean flow within the channel exactly balances that outside of the channel so that in its undisturbed state the flexible insert rests along $y=0$.

\subsection{State-space solution for a channel with a finite flexible insert}

To solve the governing equations we extend the method developed by Pitman and Lucey (2009) in which a finite-difference representation of the structural dynamics is fully coupled with a boundary-element flow solution to derive a single matrix equation from which the eigenvalues are then directly extracted. The main advantage of this method, over the traditional Galerkin method, is that it avoids a presumption that the eigenmodes of the coupled fluid-structure system can be readily assembled from a finite set of orthogonal functions that are usually the in vacuo eigenmodes of just the structure. This advantage can be especially useful for structurally inhomogeneous flexible walls for which analytical orthogonal functions are unwieldy or simply unavailable. A more complete discussion of its advantages is provided in the Pitman and Lucey (2009) paper.

The upper and lower channel walls are each discretised into $N / 2$ panels (giving the system a total of $N$ panels) and singularities, satisfying the Laplace equation, are distributed over each panel. The singularities chosen are sources and sinks as a non-lifting surface is being modelled and the strengths of the singularities are determined by the enforcement of the kinematic boundary conditions of Eq. (4) at the control point of each panel. The linearised theory developed by Lucey and Carpenter (1992) is used wherein the singularities on the deformed insert do not move with the displaced surface but stay fixed on the undisturbed plane similar to the approach of thin-aerofoil theory. This linear approximation removes the time-dependence of the influence-coefficient matrices so they need only be calculated once (for details see Lucey et al. (1997)). The boundary condition Eq. (4b) can be applied at $y=0$ when using this linear approximation.

The pressure perturbation at the mass points of the flexible insert is found using Eq. (3) in the form,

$$
-\{\triangle p\}=2 \rho_{\mathrm{f}} U^{2}\left[I^{T}\right]\left[D_{1}\right]\{\eta\}+2 \rho_{\mathrm{f}} U\left[I^{T}\right]\left[D^{+}\right]\{\dot{\eta}\}+2 \rho_{\mathrm{f}} U\left[I^{\Phi}\right]\left[D_{1}\right]\{\dot{\eta}\}+2 \rho_{\mathrm{f}}\left[I^{\Phi}\right]\left[D^{+}\right]\{\ddot{\eta}\},
$$

for the discretised system where $\left[I^{\Phi}\right]$ and $\left[I^{T}\right]$ are respectively the perturbation and tangential influence coefficient matrices, $\left[D^{+}\right]$is a spatially averaging matrix to evaluate the pressure at the mass points and $\left[D_{n}\right]$ is an $n^{\text {th }}$ order differentiation matrix. The four terms on the right-hand side of Eq. (5) are the hydrodynamic stiffness, hydrodynamic damping (middle two terms) and the hydrodynamic inertia respectively. Alternatively, Kornecki et al. (1976) respectively describe these as the forces arising from the centrifugal, Coriolis and linear (vertical) accelerations of fluid elements in the flow past the deforming wall. The latter description as a Coriolis force better indicates that the hydrodynamic-damping component of the fluid loading does not cause energy-dissipation; its principal effect is to couple the system modes. 
Eq. (1) written in finite-difference form is,

$$
\rho_{\mathrm{m}} h\{\ddot{\eta}\}+d\{\dot{\eta}\}+B\left[D_{4}\right]\{\eta\}+K\{\eta\}-T\left[D_{2}\right]\{\eta\}=-\triangle p(x, y, t),
$$

and by matching the pressure at the fluid-solid boundary using Eqs. (5) and (6), a single matrix equation is derived of the form,

$$
\{\ddot{\eta}\}=[E]\{\dot{\eta}\}+[F]\{\eta\},
$$

where,

$$
[E]=\left[-\rho_{\mathrm{m}} h[I]+2 \rho_{\mathrm{f}}\left[I^{\Phi}\right]\left[D^{+}\right]\right]^{-1}\left[d[I]-2 \rho_{\mathrm{f}} U\left[I^{\Phi}\right]\left[D_{1}\right]-2 \rho_{\mathrm{f}} U\left[I^{T}\right]\left[D^{+}\right]\right]
$$

and

$$
[F]=\left[-\rho_{\mathrm{m}} h[I]+2 \rho_{\mathrm{f}}\left[I^{\Phi}\right]\left[D^{+}\right]\right]^{-1}\left[B\left[D_{4}\right]+K[I]-T\left[D_{2}\right]-2 \rho_{\mathrm{f}} U^{2}\left[I^{T}\right]\left[D_{1}\right]\right],
$$

where $[I]$ is the identity matrix. Using standard state-space formulation, Eq. (7) can be re-written as,

$$
\{\dot{x}\}=[H]\{x\},
$$

where,

$$
\{x\}=\left\{\begin{array}{l}
\{\eta\} \\
\{\dot{\eta}\}
\end{array}\right\}
$$

and

$$
[H]=\left[\begin{array}{c}
{[0][I]} \\
{[F][E]}
\end{array}\right] .
$$

The eigenvalues $S$ and eigenvectors $W$ of this system are then extracted from $[H]$, where $S=S_{\mathrm{R}}+\mathrm{i} S_{\mathrm{I}}$ is the complex frequency of the plate, and the eigenvectors can be used to assemble the eigenmode of the flexible insert $\eta(x, t)$.

\subsection{Theoretical analysis of an infinitely long flexible insert}

In order to complement and validate the foregoing numerical approach we extend the open-flow analysis of Carpenter and Garrad (1986) of an infinitely long flexible plate with a uniformly distributed spring foundation to account for the effect of the rigid upper wall of the channel. We choose this type of flexible insert because the critical modes in its destabilisation can have much shorter wavelengths than the wall length, thus allowing its deformation to be approximated by a travelling wave with the form,

$$
\eta=\eta_{0} \exp \{i k(x-c t)\}
$$

with the flow-velocity perturbation written as,

$$
\phi=\Phi(y) \exp \{i k(x-c t)\}
$$

where $k$ is the wavenumber, $c$ is the complex wave speed and $\eta_{0}$ is the amplitude of the boundary perturbation. Use of Laplace's equation (2) gives the relation $\Phi(y)=A_{1} \exp ^{k y}+B_{1} \exp ^{-k y}$, with unknowns $A_{1}$ and $B_{1}$ that are determined by enforcing the boundary conditions in Eq. (4). Use of the Bernoulli equation, (3), applied to the streamline along $y=0$ then yields the pressure perturbation,

$$
-\Delta p=\rho_{\mathrm{f}} \eta_{0} k(U-c)^{2} \zeta \exp \{i k(x-c t)\},
$$

where

$$
\zeta=\frac{1}{1-\mathrm{e}^{-2 k H}}-\frac{1}{1-\mathrm{e}^{2 k H}},
$$


that effectively accounts for the confinement effects of the channel as compared with an open flow for which $\zeta=1$. Coupling this with the beam equation (1) at $y=0$ for a plate with no structural damping gives the following characteristic equation (dispersion relation) for the complex wave speed $c$,

$$
\left(\rho_{\mathrm{f}} k \zeta+\rho_{\mathrm{m}} h k^{2}\right) c^{2}-\left(2 \rho_{\mathrm{f}} k U \zeta\right) c+\left(\rho_{\mathrm{f}} k \zeta U^{2}-B k^{4}-K\right)=0 .
$$

Following Carpenter and Garrad (1986), the onset of divergence instability occurs when the phase speed, $c$, first becomes zero as the flow speed is increased. Thus, the flow speed for divergence onset is that for which the third $\left(c^{0}\right)$ term in Eq. (17) is zero and its minimum, or critical, value occurs for the wavenumber which satisfies,

$$
\frac{\partial\left(U^{2}\right)}{\partial k}=0
$$

where,

$$
U^{2}=\frac{B k^{4}+K}{\rho_{\mathrm{f}} k \zeta} .
$$

This minimisation yields the critical wavenumber for divergence onset, $k_{\mathrm{d}}$, and the critical flow speed for divergence is then given by,

$$
U_{\mathrm{d}}=\left(\frac{B k_{\mathrm{d}}^{4}+K}{\rho_{\mathrm{f}} k_{\mathrm{d}} \zeta}\right)^{\frac{1}{2}} .
$$

The critical wavenumber is therefore seen to depend not only on the structural parameters and fluid density but also on the channel height, $H$, through the function $\zeta$ defined by Eq. (16).

By considering the function $\zeta$, the asymptotic limits can be assessed. From Eq. (16), $\zeta \rightarrow 1$ as $H \rightarrow \infty$ and this yields,

$$
k_{\mathrm{d}}=\left(\frac{K}{3 B}\right)^{\frac{1}{4}} \quad \text { and } \quad U_{\mathrm{d}}=2\left(\frac{B K^{3}}{27 \rho_{\mathrm{f}}^{4}}\right)^{\frac{1}{8}},
$$

which recovers the results of Eq. (2.13) in Carpenter and Garrad (1986).

At a higher flow speed, modal-coalescence is known to occur when the roots of Eq. (17) are equal and so by setting the determinant equal to zero, the critical speed for modal-coalescence flutter is derived as

$$
U_{\mathrm{m}}=\left(\frac{\left(B k_{\mathrm{m}}^{4}+K\right)\left(\rho_{\mathrm{f}} \zeta+\rho_{\mathrm{m}} h k_{\mathrm{m}}\right)}{\rho_{\mathrm{f}} \rho_{\mathrm{m}} h k_{\mathrm{m}}^{2} \zeta}\right)^{\frac{1}{2}},
$$

for the critical wavenumber $k_{\mathrm{m}}$. It can be shown that if $k_{\mathrm{m}}=k_{\mathrm{d}}$ then

$$
U_{\mathrm{m}}=U_{\mathrm{d}}\left(1+\frac{\rho_{\mathrm{f}} \zeta}{\rho_{\mathrm{m}} h k_{\mathrm{d}}}\right)^{\frac{1}{2}},
$$

which agrees with Eq. (2.14) in Carpenter and Garrad (1986) in the limit $H \rightarrow \infty$ that gives $\zeta \rightarrow 1$.

To assess the effect of the channel height in the limit of very narrow channels it is possible to show that $\zeta \rightarrow 1 /(k H)$ as $H \rightarrow 0$, and this yields the divergence and modal-coalescence speeds as,

$$
U_{\mathrm{d}}=\left(\frac{H}{\rho_{\mathrm{f}}}\left(B k_{\mathrm{d}}^{4}+K\right)\right)^{\frac{1}{2}} \quad \text { and } \quad U_{\mathrm{m}}=\left(\frac{\left(B k_{\mathrm{m}}^{4}+K\right)\left(\rho_{\mathrm{f}}+\rho_{\mathrm{m}} h k_{\mathrm{m}}^{2} H\right)}{\rho_{\mathrm{f}} \rho_{\mathrm{m}} h k_{\mathrm{m}}^{2}}\right)^{\frac{1}{2}}
$$

In the results that follow we compare these analytical predictions and asymptotic forms with the results of the state-space analysis for finite flexible inserts. However, the latter analysis does not clearly distinguish between divergence and modal coalescence for a spring-backed wall. As the flow speed increases the plate's behaviour shows a transition from divergence to modal coalescence. This type of gradual transition from divergence, as opposed to an explosive onset of flutter, was also found in the numerical simulations of Lucey 
and Carpenter (1992). It is therefore difficult to compare the foregoing analytical predictions for modalcoalescence flutter onset (Eqs. (22) and (24b)) with the results of the state-space solution. Thus, we only focus on divergence-onset for spring-backed flexible plates because this instability yields the critical flow speed and is also shown to be a travelling-wave type of dynamic instability in Pitman and Lucey (2009).

\section{Results and discussion}

The results are presented using a non-dimensional scheme based upon $L$ and $L / U$ as the characteristic length and time scales. The system behaviour can then be summarised by its dependence upon three non-dimensional parameters, namely, the mass ratio

$$
\mu=\frac{\rho_{\mathrm{f}} L}{\rho_{\mathrm{m}} h}
$$

the non-dimensional stiffness ratio,

$$
\Lambda^{\mathrm{F}}=\frac{\rho_{\mathrm{f}} U^{2} L^{3}}{B}, \quad \Lambda^{\mathrm{M}}=\frac{\rho_{\mathrm{f}} U^{2} L}{T},
$$

defined respectively for simple flexible plates and tensioned membranes, and the non-dimensional channel height $H / L$. Note that the inclusion of structural damping introduces a further control parameter to the base system. For the spring-backed flexible plate, or compliant wall, $L$ no longer represents the characteristic length scale and therefore appropriate alternatives are found and discussed in $\S 3.3$. To illustrate the physical systems for which the results apply, Tab. 1 summarises the dimensional properties, and corresponding mass ratios, of the different types of flexible insert for which non-dimensional results are presented.

Before progressing, comment is made on the location of the fluid entry and exit boundary conditions and the discretisation parameters required to achieve convergence and sufficient accuracy in the numerical evaluations of the results presented. Assessment of the effect of the length chosen for rigid-channel walls up- and down-stream of the flexible insert as seen in Fig. 1 shows that for wide channels $(H / L>1.5)$ and very narrow channels $(H / L<0.1$, such as those considered in $\S 3.3)$ changing $L_{\mathrm{r}}$ does not change the eigenvalue solution and therefore in $\S 3.3$ we use $L_{\mathrm{r}}=0$. For all other channel heights as considered in $\S 3.1$ and $\S 3.2, L_{\mathrm{r}}=2 L$ is sufficiently large for the up- and down-stream rigid walls of the channel not to influence the numerical evaluations to within less than $1 \%$. With the flexible insert modelled by $N_{\mathrm{f}}$ panels (or equidistantly spaced collocation points), the upper rigid wall therefore comprises $5 N_{\mathrm{f}}$ panels so that the system has a total of $N=10 N_{\mathrm{f}}$ panels. However, for the spring-backed plate considered in $\S 3.3$ the system has a total of $N=2 N_{\mathrm{f}}$ panels.

For the simple plates and tensioned membrane used in $\S 3.1$ and $\S 3.2, N_{\mathrm{f}}=200$ provides numerical accuracy to within $0.4 \%$ while the spring-backed plate (compliant wall) requires a greater discretisation due to the higher-order mode shape of the wall. For spring stiffnesses $K=3.68 \times 10^{6}, 3.68 \times 10^{7}$ and $3.68 \times 10^{8}$ we found $N_{\mathrm{f}}=480,800$ and 1420 respectively, to be sufficient to achieve the same level $(0.4 \%)$ of numerical convergence based on the critical mode at which divergence occurs.

\subsection{Flexible plate}

Herein, the flexible insert studied has the structural dynamics of a flexible plate that, for example, could correspond to a thin aluminium panel. We first consider a dense fluid such as water. The combination of solid and fluid densities along with the plate geometry yields the mass ratio $\mu=92.3$ used to generate the non-dimensional results presented in Figs. 2 and 3. The variation with the stiffness ratio (non-dimensional flow speed) of the real and imaginary parts of the eigenvalues for two contrasting channel heights, $H / L=2$ and 0.1 , are shown in Fig. 2 (a). The real part of the eigenvalue determines the growth/decay rate of plate deflections and the imaginary part determines the oscillation frequency of each mode. Note that only the lowest two eigenvalues are plotted in this figure although all $2 N_{\mathrm{f}}$ eigenvalues of the complete fluid-structure system are calculated. Because the time-scale $L / U$ is inappropriate for plate vibrations at zero flow speed, 
the non-dimensional frequency, $S^{\prime}=S / S_{0}$, used in the results is based upon the angular oscillation frequency of the fundamental mode of the plate in vacuo, that is,

$$
S_{0}=\left(\frac{\pi}{L}\right)^{2} \sqrt{\frac{B}{\rho_{\mathrm{m}} h}} .
$$

In Fig. 2 (a), there are four zones of $\Lambda^{\mathrm{F}}$ (for each channel height) that correspond to four distinct types of plate behaviour (Pitman and Lucey, 2009). These zones are delineated here by vertical dashed lines for the $H / L=2$ case. The first is the pre-divergence zone, when the eigenvalue of the fundamental mode has an imaginary but no real part, hence the plate displays neutrally stable oscillatory motion. When the eigenvalue first has a real part, divergence sets in. For higher $\Lambda^{\mathrm{F}}$ the real part again becomes zero returning the plate to the neutrally stable oscillatory motion known as divergence recovery. The final type of behaviour with increased $\Lambda^{\mathrm{F}}$ is initiated by the coalescence of the fundamental and second modes and the plate undergoes a flutter-type motion. For the case $H / L=2$, Fig. 2 (a) shows that the transitions called divergence onset, divergence recovery and flutter onset - between these four zones occur at $\Lambda^{\mathrm{F}}=40$, 277 and 316 respectively. For the results at $H / L=2$ the eigenvalue solution agrees with that presented in Pitman and Lucey (2009) for the open-flow system which was validated against a Galerkin analysis. The present divergence-onset prediction at $\Lambda^{\mathrm{F}}=40$ also agrees with the findings of, for example, Weaver and Unny (1970), Garrad (1982), and Tan et al. (2013) thereby validating the present computational modelling and its implementation.

Figure 2 (b) shows the effect of including structural damping in the system that yielded Fig. 2 (a). For simplicity, a dashpot-type damping, seen in Eq. (1), with $d=7500 \mathrm{Ns} / \mathrm{m}^{3}$ is used. This value is chosen to facilitate appropriate comparison with the open-flow results presented in Pitman and Lucey (2009) and its value represents an amplitude attenuation of $50 \%$ per cycle of the fundamental in vacuo plate mode. As would be expected, in the pre-divergence regime of stiffness ratio, the effect of structural damping is to attenuate the modes that were neutrally stable for the elastic plate. Turning to the range of stiffness ratio that yields instability, it is evident that the inclusion of structural damping both increases the size of the divergence loop and rotates it anticlockwise about the $S_{\mathrm{R}}=0$ axis. This occurs because the dashpot-type damping exercises a greater effect at the higher modal frequencies that are obtained at lower values of $\Lambda^{\mathrm{F}}$. However, the combination of these two effects upon the divergence loop is such that for each channel height the divergence-onset stiffness ratio remains the same as for the plate with no damping; this is most evident for the case $H / L=2$ in Fig. 2 (b) for which the divergence-onset threshold has been marked with the dashed line $\Lambda^{\mathrm{F}}=40$, the same threshold shown in Fig. 2 (a). This is to be expected because divergence onset occurs as a static phenomenon and the absence of motion means that structural damping does not play a part in its determination.

At post-divergence stiffness ratios structural damping exercises significant changes in the morphology of the eigenvalue solution that are similar to those found by Pitman and Lucey (2009) for the open-flow case. Firstly, there is no divergence recovery zone, because the real parts of the fundamental and second modes are no longer symmetrical about $S_{\mathrm{R}}=0$. Secondly, instead of a distinct point at which coalescence of the oscillatory-part of the frequency occurs, there is now a gradual transition in the unstable behaviour of the plate from divergence to flutter. The same modification (as compared with that for an elastic plate) to the eigenvalue solution morphology is also found when very low levels of damping are included. These effects, and the relationship between divergence and flutter, were demonstrated and discussed in the numerical work of Lucey and Carpenter (1992).

The continuous variations with channel height of divergence-onset, divergence recovery and flutter-onset stiffness ratio, $\Lambda_{c}^{\mathrm{F}}$ (threshold flow speed for a given flexible plate and fluid), are presented in Fig. 3 (a) for both elastic and damped plates. The horizontal dashed lines indicate corresponding values for an open flow, i.e. with $H=\infty$. In the asymptotic limit of increasing channel height, the present results for channel flow approach those for an open flow and that beyond $H / L=1$ the upper channel wall has effectively ceased to have an effect on the plate's stability characteristics. Below $H / L=1$, decreasing the channel height monotonically reduces $\Lambda^{\mathrm{F}}$ for divergence onset. Divergence first occurs at the flow speed for which the plate's restorative forces exactly balance the hydrodynamic stiffness, the first term on the right-hand 
side of Eq. (5). Clearly, the magnitude of the hydrodynamic stiffness has been increased by reducing the channel height because a lower critical speed for the instability ensues. At first sight, it might appear that the increase to the hydrodynamic stiffness is caused by the plate deformation changing the effective value of $U$ in Eq. (5). However, this would be a nonlinear effect because it implies a change to the mean state of the system. In the present linear analysis, the increase to the magnitude of the hydrodynamic stiffness is caused by the effect of the upper channel wall which increases (decreases) the gradient, in the $y$-direction, of streamline curvature for a plate deformation into (out of) the channel. This occurs because the upper boundary, that has zero curvature, must always remain a streamline of the perturbed flow as it surmounts the deformation of the flexible insert in the lower channel wall.

Weaver and Païdoussis (1977) theoretically analysed a channel-flow system for inviscid flow with both walls flexible using both a Galerkin approach, where each wall was on periodic supports, and a travellingwave approach weighted for transverse curvature. In each analysis they considered the two cases of walls moving in phase (sinuous) and $180^{\circ}$ out of phase (varicose); the latter is closest to the system studied in the present work because it involves a variation to streamline curvature across the channel. Using the Galerkin analysis, Weaver and Païdoussis (1977) found that reducing the channel height caused a maximum reduction in the critical flow velocity for divergence of $29 \%$, which corresponds to approximately a $54 \%$ decrease in $\Lambda^{\mathrm{F}}$. This finding differs from the results presented herein where the critical non-dimensional flow speed for divergence reduces to zero as $H / L \rightarrow 0$. However, for this limit, our results agree with those of the travelling-wave analysis of Weaver and Païdoussis (1977). Weaver and Païdoussis (1977) also show that above $H / L=1$, in both of their theoretical models, the critical flow velocity for divergence is the same as for the open-flow case; a similar value for this threshold is demonstrated in the results of Fig. 3 (a).

Also included as an insert in Fig. 3 (a) are experimental data reported by Weaver and Païdoussis (1977) in Fig. 8 of their paper. A flattened tube was constricted, effectively creating flow between parallel flexible walls, and the flow velocity at which a flapping instability occurred, associated with divergence as opposed to flutter, was recorded for various constrictions. A best-fit line of their results was converted into our non-dimensional scheme by setting $H=0.5 b$ where $b$ was the gap between their surfaces - because they considered two flexible surfaces instead of just one - and setting our $L=0.5 \lambda$ where $\lambda$ was the observed wavelength of the flapping. Good agreement is found between our predictions and these experimental results for small $H / L$.

Figure 2 (a) also shows how channel height affects further features of the FSI system. Reducing the channel height decreases the frequency and growth/decay rate at a given flow speed. At zero stiffness ratio (zero flow speed) a lower channel height results in lower oscillation frequencies for both the fundamental and second mode, implying a higher fluid inertia, the fourth term on the right-hand side of Eq. (5). This is due to the additional $y$-constraint on the decay of fluid perturbation imposed by the upper channel wall. Thus, for narrow channels, fluid perturbations due to the flexible-plate motion extend a significant distance upstream and downstream of the moving-wall section as reflected by the need to model the rigid upstream and downstream regions, described earlier. A further consequence is that reducing the channel height reduces the initial frequency difference, at $\Lambda^{\mathrm{F}}=0$, between the fundamental and second modes. Modal-coalescence then occurs at a lower value of $\Lambda^{\mathrm{F}}$ and the amplitude of the divergence loop becomes smaller. Thus, while reducing the channel height leads to a reduction in the critical flow speed for divergence onset, beyond that threshold both the range of flow speeds over which divergence occurs and its growth rate are reduced. However, the more violent flutter instability beyond divergence sets in at a lower flow speed and frequency. The effect, as a continuous variation, of the upper channel-wall height on the first and second mode frequency at zero flow speed is shown in Fig. 3 (b) along with the frequency at which modal-coalescence occurs. These results suggest that channel-height may have a significant effect on transient growth within the system through non-normality because Coppola and de Luca (2010) show that the frequency spacing between the lowest two modes is directly linked to the beating frequency of transient growth.

All of the foregoing results pertain to the mass ratio $\mu=92.3$. We now present the effect of channel height on the system behaviour for two lower values, $\mu=9.23$ and $\mu=0.923$, using the physical data seen in Tab. 1; the latter case closely represents air flow past an aluminium plate. Fig. 4 (a) shows the eigenvalue solutions at a channel height $H / L=0.1$ for each of $\mu=9.23$ and 0.923 . For $\mu=9.23$ the solution morphology is seen to be the same as that in Fig. 2 (a). The continuous variation of divergence-onset, 
divergence-recovery and flutter-onset threshold values, $\Lambda_{c}^{\mathrm{F}}$, with channel height are presented in Fig. 4 (b) and are seen to follow very similar trends to the corresponding results for an undamped plate with $\mu=92.3$ in Fig. 3 (a). This finding agrees with Weaver and Païdoussis (1977) who concluded that the overall effect of the upper-channel wall proximity is largely unchanged by the mass ratio.

In contrast, at the mass ratio $\mu=0.923$ in Fig. 4 (a), a change to solution morphology has occurred. While the critical mode continues to be Mode-1 divergence with its onset stiffness ratio unchanged (since the instability is static exactly at onset) divergence recovery does not occur with increasing $\Lambda^{\mathrm{F}}$, but instead Mode-2 divergence occurs and, with further increases, the two divergence modes coalesce to give flutter. The quantitative summary of the respective threshold values as they vary with channel height is presented in Fig. 4 (b). This different sequence of instability transitions has been presented and its physical causes explained in Tan et al. (2013) for the corresponding open-flow configuration. What can be concluded from the new results of the present paper is that reducing the channel height does not modify the special solution morphology that applies at very low mass ratios.

In summary, the present results show that a finite flexible plate comprising one wall of an inviscid channel flow has the same eigenvalue solution morphology as for the corresponding open flow but that the proximity of the upper wall causes the instabilities of divergence and modal-coalescence flutter to occur at lower flow velocities and flutter with a lower oscillation frequency. These effects have been definitively quantified using a non-dimensional framework. The influence of the upper-wall starts to becomes significant for $H / L<1$, i.e. of the order of wall-deformation wavelength, irrespective of variations to the system's mass ratio or the inclusion of structural damping.

\subsection{Tensioned membrane}

To complete the investigation of flexible inserts comprising a single structural component, for which the lowest-order modes of deformation based on insert length give the critical values at destabilisation, we consider a tensioned elastic membrane with mass ratio $\mu=92.3$. This type of insert has more relevance to applications in biomechanics than the preceding flexible-plate systems.

Figure 5 (a) displays the dependence of system eigenvalues on the stiffness ratio (non-dimensional flow speed), $\Lambda^{\mathrm{M}}$, defined using the membrane tension as seen in Eq. (26b). The frequencies are now nondimensionalised by the angular oscillation frequency of the fundamental mode of the membrane in vacuo,

$$
S_{0}=\left(\frac{\pi}{L}\right) \sqrt{\frac{T}{\rho_{\mathrm{m}} h}} .
$$

The eigenvalue solution is qualitatively similar to that of the simple plate presented in $\S 3.1$, the physical interpretation of which, discussed therein, carries across to the FSI phenomenology of the tensioned membrane. Although divergence, divergence recovery and flutter now occur at much lower values of the stiffness ratio, the dimensional flow speeds at which instabilities set in is within the same order of magnitude for the illustrative physical data given in Tab. 1. The interaction of a tensioned membrane with viscous channel flow has received significant attention. Studies such as those of Luo and Pedley (1996), Luo and Pedley (2000), Huang (2001) and Jensen and Heil (2003) model unsteady laminar flow and (with the exception of Huang (2001)) large amplitude deformations. Therefore, direct comparison with the present results that use ideal flow can only be at a very broad level. What the two types of system are seen to have in common is that reductions to the membrane tension - that increase the value $\Lambda^{\mathrm{M}}$ in the present work - cause the onset of instability to occur at a lower flow speed or Reynolds number. In studies of viscous flow, the channel height determines the Reynolds number and thus its reduction would be stabilising because the overall balance between inertial forces and viscous forces changes in favour of the latter. In the present system the opposite is true because the destabilising inertial forces are intensified by reductions to the channel height with no ameliorating viscous effects. This effect is summarised in Fig. 5 (b). It is noted that the absence of viscous effects in the present modelling renders the results non-physical for extremely low values of $H / L$. As a landmark, below $H / L=0.003$ the dimensional data in Tab. 1 would yield Reynolds numbers in the laminar range of flow speeds, when using the kinematic viscosity of water at $20^{\circ} \mathrm{C}$ and the flow speed at divergence-onset as the characteristic speed; this limitation also applies to the flexible-plate cases analysed 
in $\S 3.1$. Finally, in common with the flexible-plate results of Fig. 3 (a), the effects of channel height become insignificant for $H / L$ greater than 1 , beyond which the system behaves identically to that of a tensioned membrane subjected to an open-flow.

\subsection{Spring-backed flexible plate}

The stability of a flexible plate supported by a uniformly distributed spring foundation is now considered. The introduction of a further structural component - the spring backing - creates significantly different stability characteristics because, for example, divergence onset is now determined by the combination of plate flexure, spring stiffness and the hydrodynamic-stiffness fluid loading. In particular, it is no longer the low-order modes having wavelengths of the order of the plate length that are the first to be destabilised as found in $\S 3.1$ and $\S 3.2$. Accordingly, the plate length, $L$, is not a suitable characteristic length for the FSI system and the following non-dimensional scheme, based upon a local characteristic length $\left(\rho_{\mathrm{m}} h / \rho_{\mathrm{f}}\right)$, is introduced following Lucey and Peake (2003) wherein

$$
H^{\prime}=\frac{H \rho_{\mathrm{f}}}{\rho_{\mathrm{m}} h}, \quad U^{\prime}=\frac{U\left(\rho_{\mathrm{m}} h\right)^{3 / 2}}{\rho_{\mathrm{f}} B^{1 / 2}} \quad \text { and } \quad K^{\prime}=\frac{K\left(\rho_{\mathrm{m}} h\right)^{4}}{B} .
$$

Thus, Eqs. (29a, b) replace the control parameters defined by Eqs. (25) and (26), while the spring foundation introduces the additional control parameter $K^{\prime}$. The length of the flexible insert, $L$, contributes the further control parameter, $L^{\prime}=L \rho_{\mathrm{f}} /\left(\rho_{\mathrm{m}} h\right)$, but it will be seen this has only a marginal influence on system response for the cases studied herein where the wavelength of the critical mode is much shorter than the panel length, a situation promoted by increases either to insert length or the stiffness of the spring foundation.

Figures 6 (a) and (b) respectively show the dependence of the lowest 40 eigenvalues of the FSI system on the flow speed for the equivalent open flow and for a channel with $H^{\prime}=0.7$ when $K^{\prime}=4.367$. The illustrative physical data for this system given in Tab. 1 are close to those of the compliant walls investigated by Carpenter and Garrad (1986), Lucey et al. (1997) and Pitman and Lucey (2009) that were chosen to represent water interacting with the compliant coating of Kramer (1960) that showed the best performance for transition postponement in an open boundary-layer flow. In these figures the onset of divergence occurs when the real part of an eigenvalue first becomes positive; the flow speed at which this occurs is marked in each of Figs. 6 (a) and (b) by a vertical dashed line. For the open-flow case $\left(H^{\prime}=\infty\right)$, divergence-onset occurs at $U^{\prime}=2.34$. By plotting the associated eigenvector, or tracing the mode that first crosses the $S_{\mathrm{I}}=0$ axis in Fig. 6 (a), the critical mode is found to be 24, giving the critical wavelength, $\lambda_{\mathrm{s}}^{\prime}=5.87$ where $\lambda_{\mathrm{s}}^{\prime}=\lambda_{\mathrm{s}} \rho_{\mathrm{f}} /\left(h \rho_{\mathrm{m}}\right)$. These critical values agree well with the predictions of $U^{\prime}=2.30$ and $\lambda_{\mathrm{s}}^{\prime}=5.72$, given by Eqs. (21) that recovered the analytical expressions of Carpenter and Garrad (1986) for a corresponding compliant wall of infinite length. It is remarked that $\lambda_{\mathrm{s}}^{\prime} / L^{\prime}=0.0834$ for the critical mode arising at divergence onset in Fig. 6 (a). Thus, at this very small value of the ratio of disturbance wavelength to insert length, the actual length of the flexible insert has little influence on the critical mode and flow speed for divergence onset. At flow speeds just above divergence onset, Pitman and Lucey (2009) showed that divergence instability is realised as slow downstream-travelling amplifying waves, a form that is predicted by a travelling-wave theory based upon a wall of infinite extent. This realisation of divergence instability as slow downstream-travelling waves continues to hold in the present channel-flow system.

Comparing the result of Fig. 6 (b) with that of Fig. 6 (a) it is seen that finite channel height reduces the critical flow speed for divergence onset although the solution morphology of the eigen-system is largely unchanged. The critical mode now has a wavelength $\lambda_{\mathrm{s}}^{\prime}=6.12$ which is longer than that of the corresponding open-flow case. The variation of divergence-onset flow speed with channel height is summarised in Fig. 7 (a) for the parameters used to generate Fig. 6 and two further levels of foundation spring-stiffness, $K^{\prime}$. Also included in this figure are the analytical predictions of Eq. (20) based upon the assumption of an infinitely long flexible insert for each of the spring foundations. As would be expected, the lines of critical flow speed are displaced upwards for higher values of $K^{\prime}$; a stiffer wall has a higher critical speed. It is also noted that the wavelength of the critical mode becomes shorter as $K^{\prime}$ is increased. For each of the pairs of lines associated with a $K^{\prime}$, the effect of reducing the channel height is seen to be destabilising and this effect begins to occur below values of $H^{\prime}$ that are lower when $K^{\prime}$ is higher. Thus, the effect of channel height 
scales with the wavelength of the critical mode. This suggests that a general result for all spring-backed flexible plates is attainable. We therefore define channel height relative to critical wavelength, $H / \lambda_{\mathrm{s}}$, and non-dimensionalise the flow speed as a stiffness ratio defined as,

$$
\Lambda^{\mathrm{I}}=\frac{\rho_{\mathrm{f}} U^{2}}{\left(B K^{3}\right)^{1 / 4}},
$$

which is based upon the length scale and flow-to-structural pressure ratio implicit in Eqs. (21a, b) and discussed in Lucey et al. (1997). Re-plotting all of the data in Fig. 7 (a) gives the result of Fig. 7 (b) in which the different $K^{\prime}$-curves have collapsed onto a single variation of divergence-onset flow speed with channel height.

Having definitively characterised the divergence-onset results from the state-space analysis of an insert with finite length, albeit long relative to the wavelength of the critical mode, attention is now given to the validity of our analytical prediction based upon the assumption of an insert of infinite length. The latter is plotted in Fig. 7 (b) along with the results of the asymptotic limits, $H \rightarrow 0$ and $H \rightarrow \infty$, given by the non-dimensionalised forms of Eqs. (21b) and (24a) respectively. The two methods clearly agree well and we note that the percentage difference between the two methods (being $2.43 \%$ for $H / \lambda_{\mathrm{s}}>0.6$ ) reduces with the channel height so that, for example, at $H / \lambda_{\mathrm{s}}=0.02$ there is a $0.2 \%$ difference. The simple asymptotic forms, Eqs. (21b) and (24a), are seen to yield excellent agreement with the results of full theoretical treatments over a surprisingly wide range of each of the low and high values $H / \lambda_{c}$ for which they are solutions in the exact limit.

To explain why there is better agreement as the channel is made narrower, Fig. 8 (a) compares insert deformations for the case $K^{\prime}=4.367$ and channel height $H^{\prime}=2.0$ (effectively an open flow) at divergence onset $\left(U^{\prime}=2.3\right)$ as assumed in the travelling-wave solution (upper panel) and as calculated using the statespace solution (lower panel). It is evident that while both support a critical mode with the same wavelength, the latter features an amplitude modulation the shape of which is very close to that of the fundamental mode that has been sketched in using a dashed line. Amplitude modulation was shown in the divergence waves predicted by Pitman and Lucey (2009) for an open flow at flow speeds higher than that of divergence-onset; their results also identify unstable states where the amplitude modulation takes the shape of the second and third modes of the overall panel. However, it is most likely that the lowest energy state - that of the fundamental - would dominate the physical destabilisation of the finite flexible wall. Fig. 8 (b) makes the same comparison as Fig. 8 (a) but now for channel flow with $H^{\prime}=0.5$, again exactly at divergence onset $\left(U^{\prime}=1.47\right)$. For the finite wall (lower panel) there is now little evidence of amplitude modulation in the calculated deformation and therefore the form assumed in the travelling-wave assumption of disturbances (upper panel) yields results that more closely agree with the exact representation of the compliant insert.

To demonstrate how the condition of finiteness affects the hydrodynamic loading, Fig. 8 (c) shows the spatial variation of the coefficient of the hydrodynamic stiffness term in Eq. (5) for a uniform deformation amplitude, as assumed in the analysis of a flexible wall of infinite length, for each of $H^{\prime}=0.5, H^{\prime}=2.0$ and the corresponding open flow each at the flow speed of divergence onset. Clearly the pressure evaluation based upon the assumption of an infinite flexible wall (not plotted) would yield a constant value across the wall for each case in Fig. 8 (c). For the finite-wall pressure evaluation, the pressure loading is seen to be constant across the middle region of flexible wall but there is significant variation adjacent to the leading and trailing edges of the flexible panel. This reflects the fact that for a finite insert, each spatial location is unique with respect to the hydrodynamic influence it receives from the effect of boundary deformations upstream and downstream of it. For open flows, a discussion, and mathematical demonstration, of the spatial variation of hydrodynamic forces over a compliant wall of finite length is presented in Lucey and Carpenter (1993) and the special modelling required to model finite panels using travelling-wave approaches is presented in Peake (2004). It is the imbalance between structural forces and pressure loading for uniform amplitude that leads to amplitude modulation - the state in which these forces are exactly balanced across the entire flexible wall - when a finite wall is correctly analysed. However, what the results Fig. 8 (c) clearly show is that as the channel height is reduced, the extent of the influence of the leading- and trailing-edge effects on the pressure reduces and therefore the assumptions in the travelling-wave model are less restrictive 
in its representation of a truly finite compliant wall interacting with an inviscid fluid flow.

Finally, the present travelling-wave analysis has been extended to model a channel having both lower and upper spring-backed flexible plates so as to compare our predictions with the flexible-channel analysis of Weaver and Païdoussis (1977). When both walls of the channel are flexible, the results show close qualitative agreement with the results of Weaver and Païdoussis (1977) that were obtained using a travelling-wave assumption for the stream-wise variation of wall deformations combined with a modal weighting to account for transverse three-dimensional effects. Exact quantitative agreement could not be expected because of the transverse effects in Weaver and Païdoussis (1977) that increase the divergence-onset flow speed as compared with a two-dimensional analysis and because in the present study a spring-backed flexible-plate is used. These results (being similar to those presented in Weaver and Païdoussis (1977), but not presented here) are mentioned as a further source of validation. When the channel height for the two-sided flexible insert is twice that used in the analysis of a one-sided flexible insert exact collapse of results occurs showing that the straight channel centreline of the former has the same effect as the flat upper wall in the one-sided spring-backed flexible-plate case.

The present investigation of a spring-backed flexible plate as one wall of a fluid-conveying plane channel has resulted in the characterisation of divergence-onset as a single variation of non-dimensional critical flow speed with channel height that accounts for all of the system's physical parameters. This definitive quantification shows that reducing the channel height has a destabilising effect, in that it reduces the critical flow speed, and that channel-height effects scale with the wavelength of the critical divergence mode. The analytical treatment, based upon a travelling-wave assumption of disturbance form, also developed in this paper, is shown to agree well with the predictions of our state-space solution that correctly accounts for the finiteness of the flexible insert. The relatively small differences in the predictions of the two methods developed in this paper has been explained as arising from amplitude modulation of the critical mode when fixed leading- and trailing-edge are strictly enforced. However this disparity has been shown to reduce as channel height is decreased. Finally, simple asymptotic forms of the full analytical solution have been shown to provide excellent approximations of critical speed over significant ranges of both small and large channel heights.

\section{Conclusion}

An extension of the modelling of Pitman and Lucey (2009) that comprises a hybrid of computational and theoretical methods has been developed to study the stability of a flexible insert in one wall of an otherwise rigid channel conveying an inviscid flow. Results for the case where the upper channel wall is sufficiently far from the lower wall in which the flexible insert is embedded recover the results of previous studies of the classical problem of a flexible panel or compliant wall interacting with an open flow. For flexible inserts comprising a structure for which the critical mode at instability onset is much shorter than the panel length, an analytical solution has been developed. In the limit of infinite channel-wall separation, this solution recovers the equivalent formulae derived for an open flow. These limiting cases serve to validate each of the two approaches developed in this paper that account for the confinement effects of finite channel height.

A comprehensive investigation of the stability of a range of flexible-insert types has been conducted using an eigen-analysis of the fluid-structure system. In all cases it was found that reducing the channel height causes the onset of divergence and modal-coalescence flutter to occur at lower flow velocities and the frequency of both system modes prior to instability onset and flutter to be reduced. These effects have been quantified in non-dimensional form. For simple plates and membranes, there is a channel height-to-insertlength, $H / L$, typically unity, above which the effects of the upper wall are negligible irrespective of the mass ratio of the FSI system and whether structural damping is included.

For the more complex structure of a spring-backed flexible plate (or compliant wall), it is found that the effect of the channel height scales with the wavelength of the critical mode as opposed to the overall length of the flexible insert. When comparing the two methods developed and deployed for this type of flexible wall, it has been shown that the analytical solution based upon a travelling-wave form of wall deformation, predicts divergence-onset flow speeds that agree very well with the corresponding analysis of a finite insert using the state-space solution. The agreement improves as the channel height is decreased. This has been 
shown to arise from a reduction, as the channel height is decreased, in the amplitude modulation of the divergence mode that occurs when fixed insert ends are modelled. This finding suggests that the classical hydrodynamic-stability approach used to make more complete fluid-flow models tractable, for example when perturbations to mean flow velocity profile arising from viscous effects are modelled, will provide a good approximation of compliant-wall interactions if the channel height is small. Simple asymptotic forms of the full analytical solution have been shown to provide excellent approximations of critical speed over appropriate ranges of channel height and these can serve as useful design formulae in engineering applications.

The overall contribution of the present paper is a comprehensive set of stability bounds for inviscid channel flow interacting with a flexible insert. These results effectively represent the FSI of flexible inserts in an otherwise rigid-walled channel at the limit of infinite Reynolds number and therefore provide useful engineering approximations for applications wherein the Reynolds number of the flow is very high. They can also serve as a benchmark - as the infinite Reynolds-number limit - for the validation of future theoretical and computational models wherein turbulent flow at finite Reynolds number interacts with and destabilises a compliant insert.

\section{References}

Bertram, C. D., Castles, R. J., 1999. Flow limitation in uniform thick-walled collapsible tubes. Journal of Fluids and Structures $13,399-418$.

Bertram, C. D., Elliott, N. S. J., 2003. Flow-rate limitation in a uniform thin-walled collapsible tube, with comparison to a uniform thick-walled tube and a tube of tapering thickness. Journal of Fluids and Structures 17, 541-559.

Bertram, C. D., Raymond, C. J., Pedley, T. J., 1990. Mapping of instabilities for flow through collapsed tubes of differing length. Journal of Fluids and Structures 4, 125-153.

Bisplinghoff, R. L., Ashley, H., Halfman, R. L., 1955. Aeroelasticity. Addison-Wesley Publishing Company, reprinted by Dover Publications in 1996.

Carpenter, P. W., Davies, C., Lucey, A. D., 2000. Hydrodynamics and compliant walls: Does the dolphin have a secret? Current Science 79, 9-13.

Carpenter, P. W., Garrad, A. D., 1985. The hydrodynamic stability of flow over Kramer-type compliant surfaces. Part 1. Tollmien-Schichting instabilities. Journal of Fluid Mechanics 155, 465-510.

Carpenter, P. W., Garrad, A. D., 1986. The hydrodynamic stability of flow over Kramer-type compliant surfaces. Part 2. flow-induced surface instabilities. Journal of Fluid Mechanics 170, 199-232.

Conrad, W. A., 1969. Pressure-flow relationships in collapsible tubes. IEEE Transactions in Biomedical Engineering BME-16, $284-295$.

Coppola, G., de Luca, L., 2010. Non-modal dynamics before flow-induced instability in fluid-structure interactions. Journal of Sound and Vibration 329(7), 848-865.

Davies, C., Carpenter, P. W., 1997. Instabilities in a plane channel flow between compliant walls. Journal of Fluid Mechanics $352,205-234$.

de Langre, E., Ouvrard, A. E., 1999. Absolute and convective bending instabilities in fluid-conveying pipes. Journal of Fluids and Structures 13, 663-680.

Doaré, O., de Langre, E., 2002. Local and global instability of fluid-conveying pipes on elastic foundations. Journal of Fluids and Structures 16, 1-14.

Dodds, H. L., Runyan, H. L., 1965. Effect of high-velocity fluid flow on the bending vibrations and static divergence of a simply supported pipe. NASA Technical Note NASA TN D-2870.

Dugundji, J., Dowell, E., Perkin, B., 1963. Subsonic flutter of panels on continuous elastic foundations. AIAA Journal 1 (5), $1146-1154$.

Garrad, A. D. \& Carpenter, P. W., 1982. A theoretical investigation of flow-induces instabilities in compliant coatings. Journal of Sound and Vibrations 84(4), 483-500.

Gavriely, N. D., Shee, T. R., Cugell, W., Grotberg, J. B., 1989. Flutter in flow-limited collapsible tubes: a mechanism for generation of wheezes. Journal of Applied Phsiology 66, 2251-2261.

Grotberg, J. B., Jensen, O. E., 2004. Biofluid mechanics in flexible tubes. Annual Review of Fluid Mechanics 36, $121-147$.

Heil, M., Hazel, A. L., 2011. Fluid-structure interaction in internal physiological flows. Annual Review of Fluid Mechanics 43, 141-162.

Hémon, P., de Langre, E., Schmid, P., 2006. Experimental evidence of transient growth of energy before airfoil flutter. Journal of Fluids and Structures 22(3), 391-400.

Huang, L., 2001. Viscous flutter of a finite elastic membrane in Poiseuille flow. Journal of Fluids and Structures 15, $1061-1088$.

Jensen, O. E., Heil, M., 2003. High-frequency self-excited oscillations in a collapsible-channel flow. Journal of Fluid Mechanics 481, 235-268.

Kornecki, A., Dowell, E. H., Brien, J., 1976. On the aerooelastic stability of two-dimensional panels in uniform incompressible flow. Journal of Sound and Vibrations 47(2), 163-178.

Kramer, M., 1960. Boundary layer stabilization by distributed damping. Journal of American Society of Naval Engineers 72 , $25-33$. 
Lucey, A. D., Cafolla, G. J., Carpenter, P. W., Yang, M., 1997. The nonlinear hydroelastic behaviour of flexible walls. Journal of Fluids and Structures 11, 717-744.

Lucey, A. D., Carpenter, P. W., 1992. A numerical simulation of the interaction of a compliant wall and inviscid flow. Journal of Fluid Mechanics 234, 121-146.

Lucey, A. D., Carpenter, P. W., 1993. On the difference between the hydroelastic instability of infinite and very long compliant panels. Journal of Sound and Vibration 163 (1), 176-181.

Lucey, A. D., Peake, N., 2003. Wave excitation on flexible walls in the presence of a fluid flow. IUTAM: Flow through collapsible tubes and past other highly compliant boundaries (Eds. P.W. Carpenter \& T.J. Pedley), 118-145.

Luo, X. Y., Pedley, T. J., 1996. A numerical simulation of unsteady flow in a two-dimensional collapsible channel. Journal of Fluid Mechanics 314, 191-225.

Luo, X. Y., Pedley, T. J., 2000. Multiple solutions and flow limitation in collapisble channel flows. Journal of Fluid Mechanics 420, 301-324.

Païdoussis, M. P., 1998. Fluid-Structure Interaction Volume 1. Academic Press, 525 B Street, Suite 1900, San Diego, California 92101-4495, USA.

Païdoussis, M. P., 2003. Fluid-Structure Interactions Volume 2. Elsevier Academic Press, 84 Theobald's Road, London WC1X 8RR, UK.

Peake, N., 2004. On the unsteady motion of a long fluid-loaded elastic plate with mean flow. Journal of Fluid Mechanics 507, $335-366$.

Pitman, M. W., Lucey, A. D., 2009. On the direct determination of the eigenmodes of finite flow-structure system. Proceedings of the Royal Society 465, 257-281.

Schmid, P. J., de Langre, E., 2003. Transient growth before coupled-mode flutter. ASME Journal of Applied Mechanics 70, 894-901.

Schwartz, M., Manzoor, S., Hémon, P., de Langre, E., 2009. By-pass transition to airfoil flutter by transient growth due to gust impulse. Journal of Fluids and Structures 25(8), 1272-1281.

Tan, B. H., Lucey, A. D., Howell, R. M., 2013. Aero-/hydro-elastic stability of flexible panels: Prediction and control using localised spring support. Journal of Sound and Vibration 322, 7033-7054.

Tsigklifis, K., Lucey, A. D., 2013. Modelling and analysis of the global stability of Blasius boundary-layer flow interacting with a compliant wall. Piantadosi, J., Anderssen, R.S. and Boland J. (eds) MODSIM2013, 20th International Congress on Modelling and Simulation. Modelling and Simulation Society of Australia and New Zealand, 775-781.

Weaver, D. S., Païdoussis, M. P., 1977. On collapse and flutter phenomena in thin tubes conveying fluid. Journal of Sound and Vibration 50, 117-132.

Weaver, D. S., Unny, T. E., 1970. The hydroelastic stability of a flat plate. ASME: Journal of Applied Mechanics $37,823-827$. 


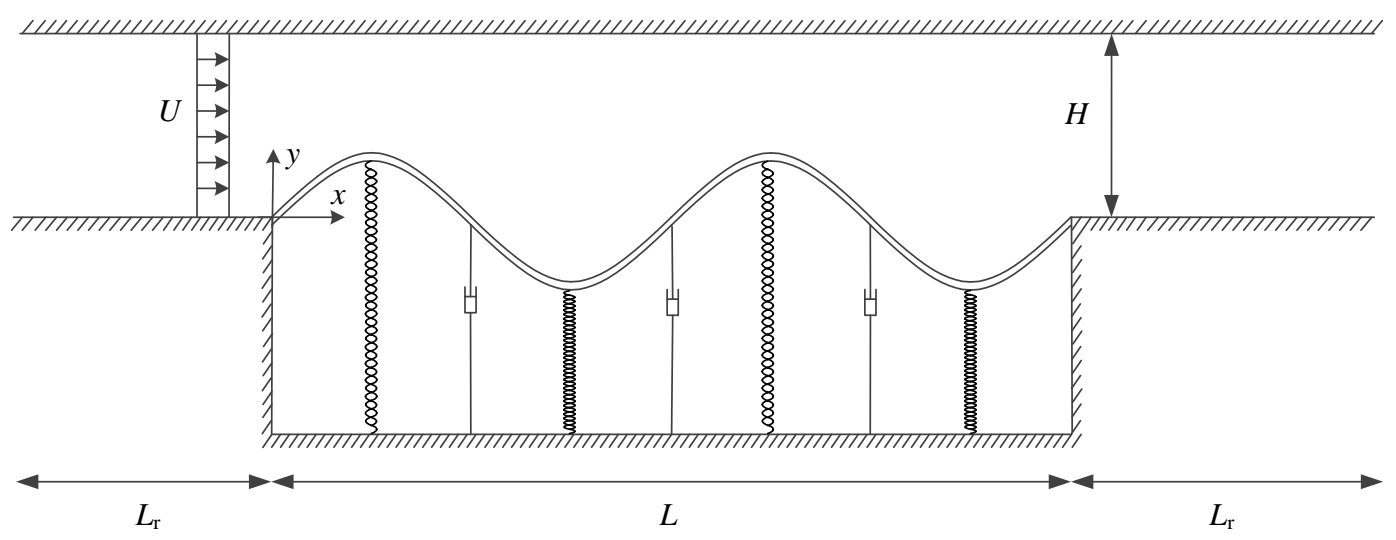

Figure 1: Schematic of the fluid-structure interaction system studied; in this illustration the insert comprises a spring-backed flexible plate (compliant wall).

(a)
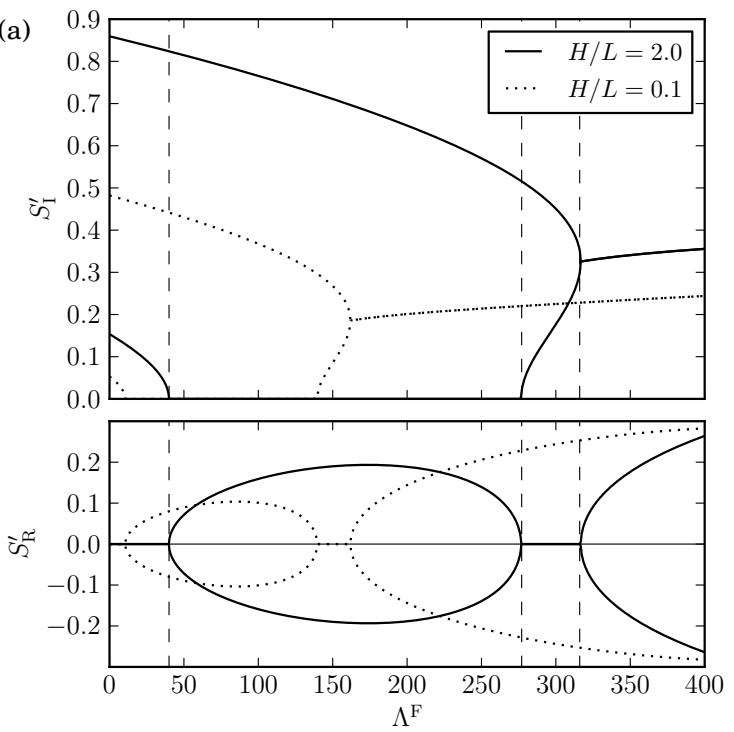

(b)
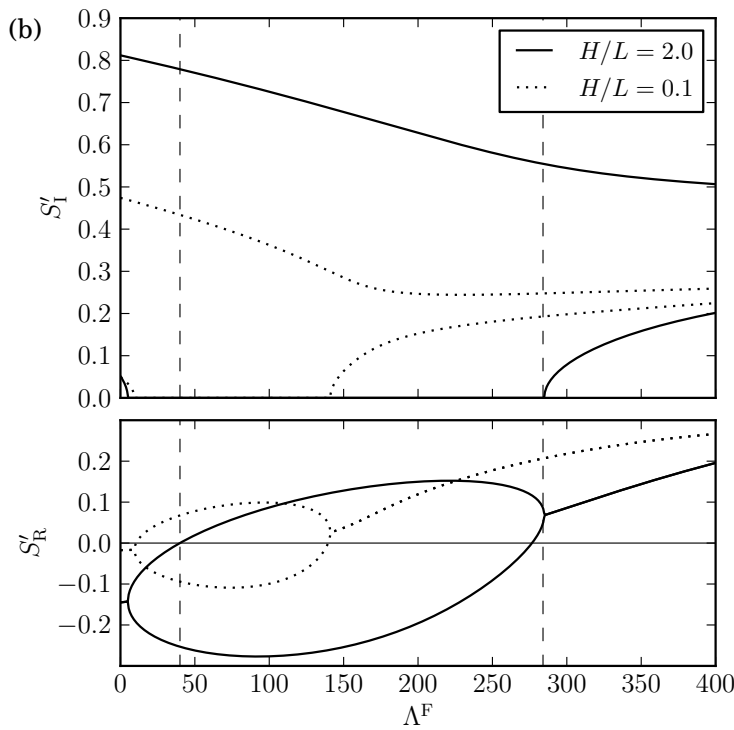

Figure 2: Variation of the real (growth/decay) and imaginary (oscillatory) parts of the first two eigenvalues with stiffness ratio (non-dimensional flow speed) for two non-dimensional channel heights for mass ratio $\mu=92.3$ : (a) Elastic plate, and (b) Flexible plate with structural damping. In each of (a) and (b) the vertical dashed lines indicate divergence onset, divergence recovery and flutter onset for the case $H / L=2$. 

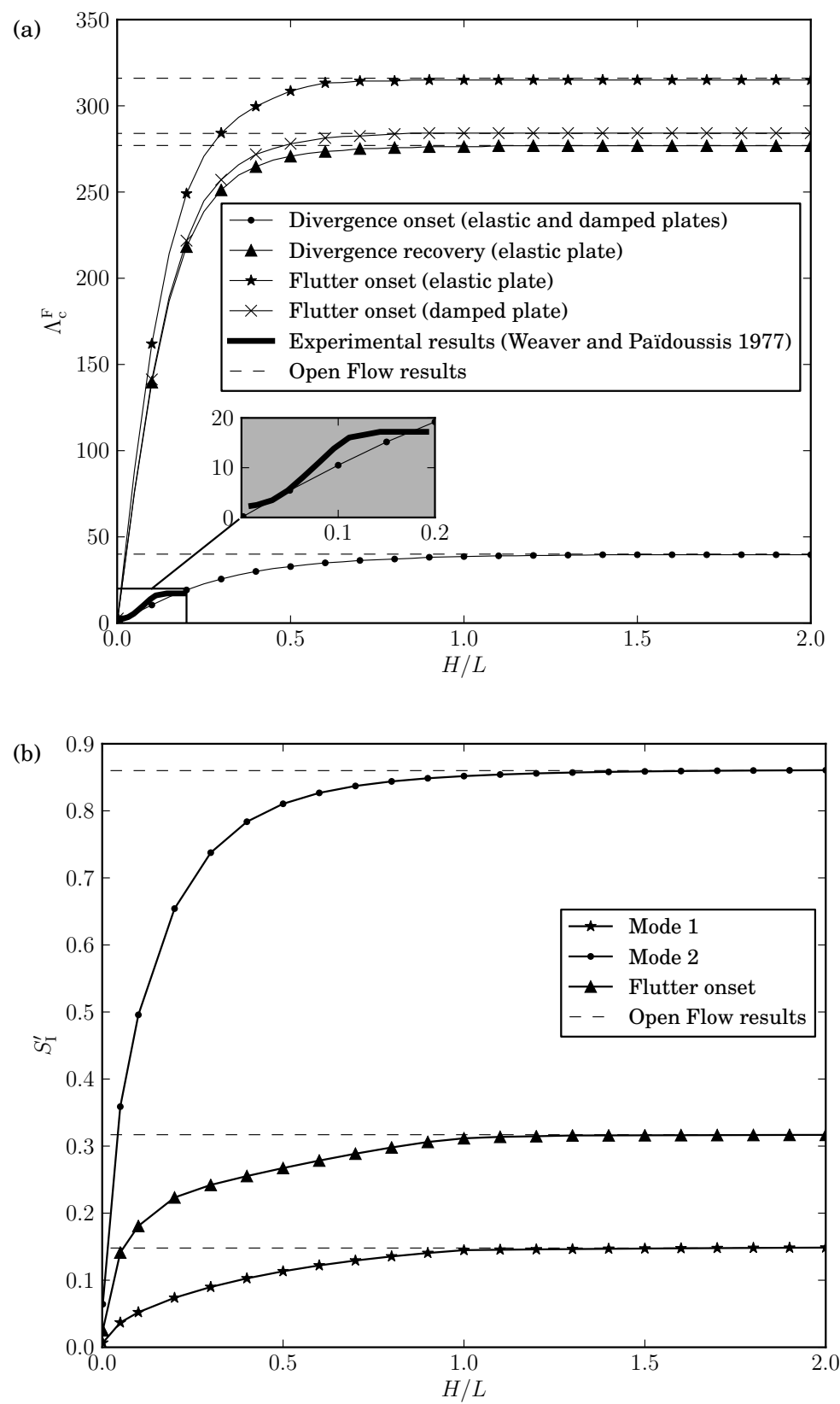

Figure 3: The effect of channel height on the FSI of a flexible plate for $\mu=92.3$ : (a) Variation of stiffness-ratio values at instability onset or recovery with channel height, and (b) The variation with channel height of Mode 1 and Mode 2 oscillation frequency at zero flow speed and modal-coalescence flutter frequency at onset. In both (a) and (b), the dashed lines indicate values for the equivalent open flow. 

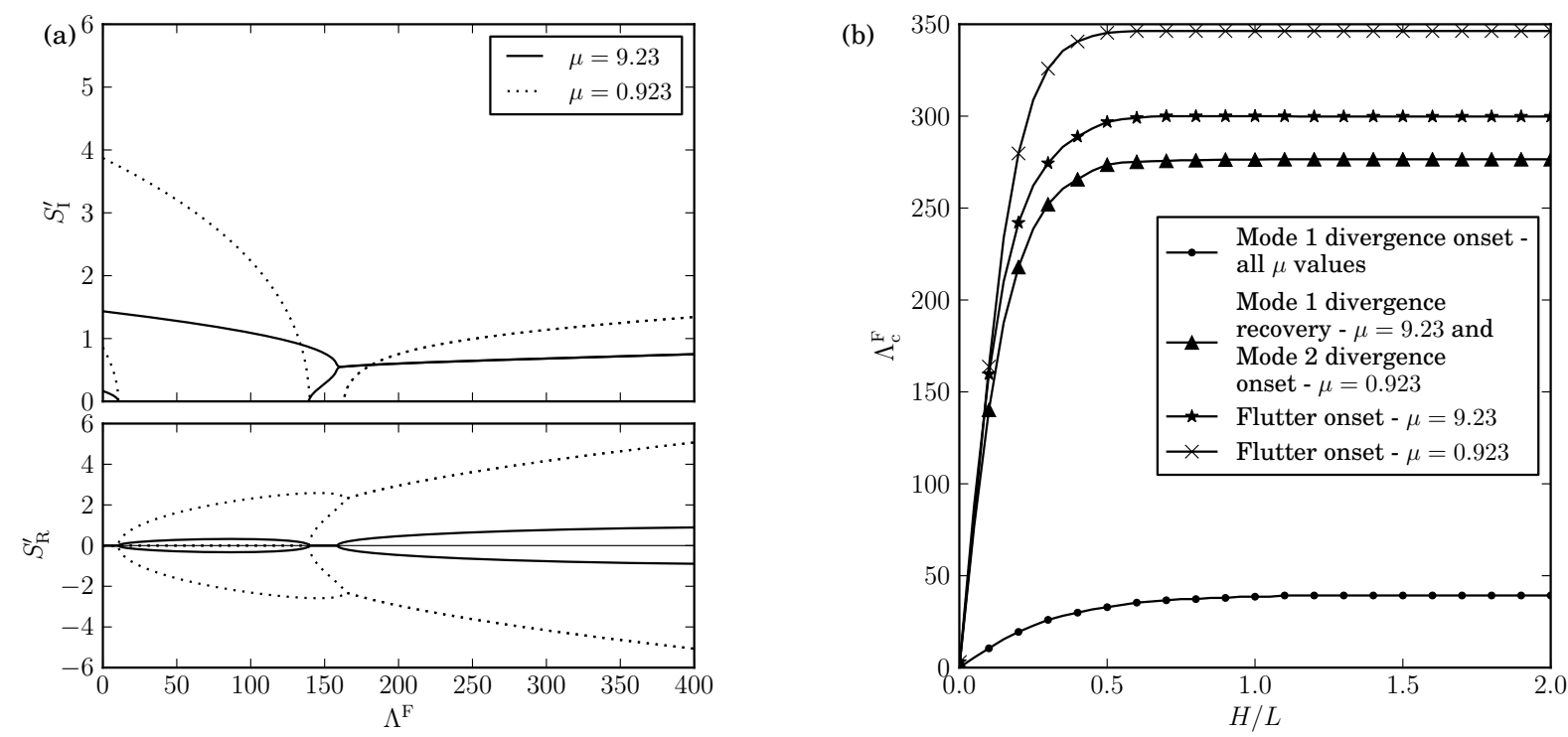

Figure 4: The effect of channel height on the stability of an elastic flexible plate for different mass ratios, $\mu$ : (a) Real and imaginary parts of the first two eigenvalues as a function of stiffness ratio (non-dimensional flow speed) at $H / L=0.1$, and (b) Variation of stiffness-ratio values at instability onset or recovery with channel height.
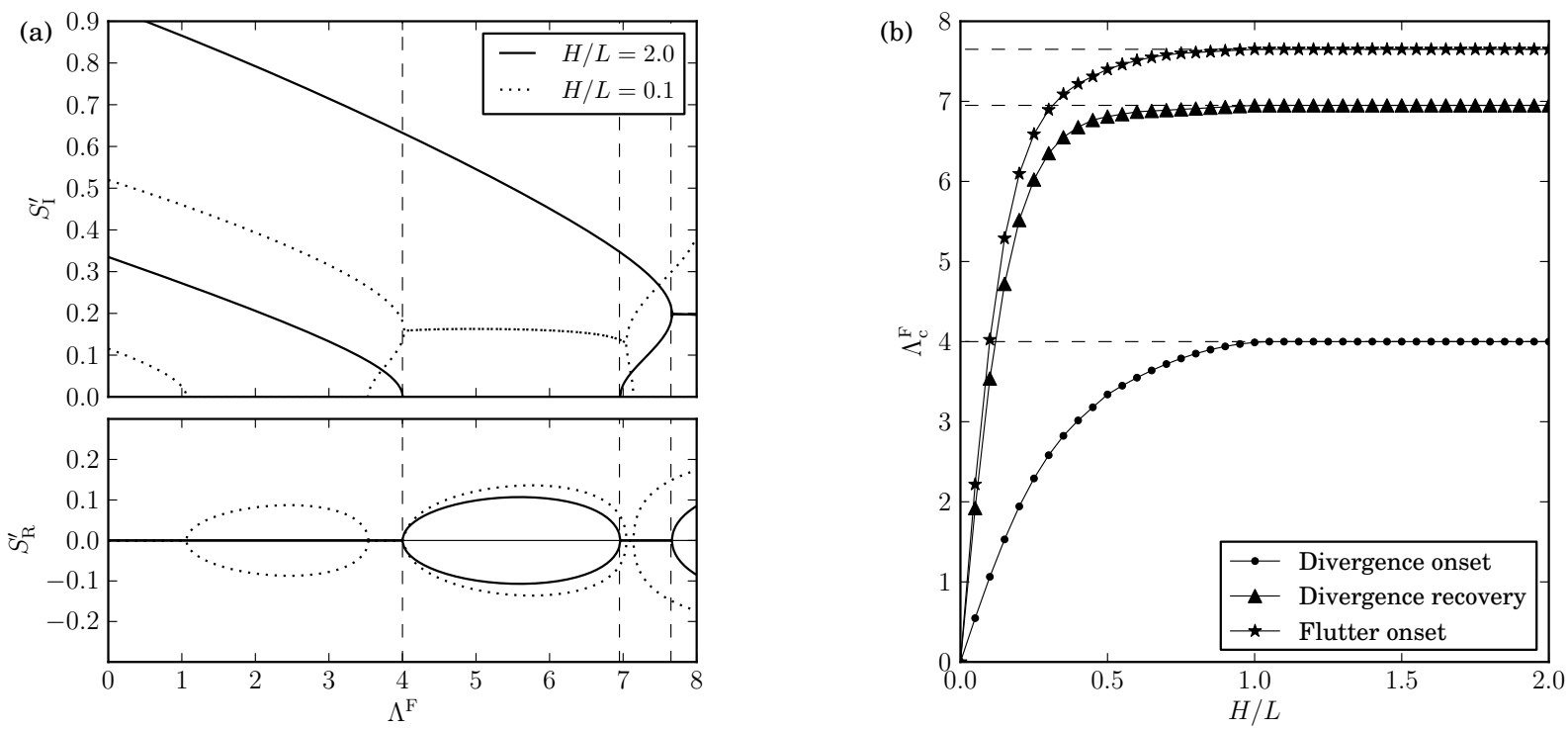

Figure 5: The effect of channel height on the stability of a tensioned membrane with mass ratio $\mu=92.3$ : (a) Variation of the real (growth/decay) and imaginary (oscillatory) parts of the first two eigenvalues with stiffness ratio (non-dimensional flow speed) for two non-dimensional channel heights; the vertical dashed lines indicate divergence onset, divergence recovery and flutter onset for the case $H / L=2$, and (b) Variation of stiffness-ratio values at instability onset or recovery with channel height; the dashed lines indicate values for the equivalent open flow. 

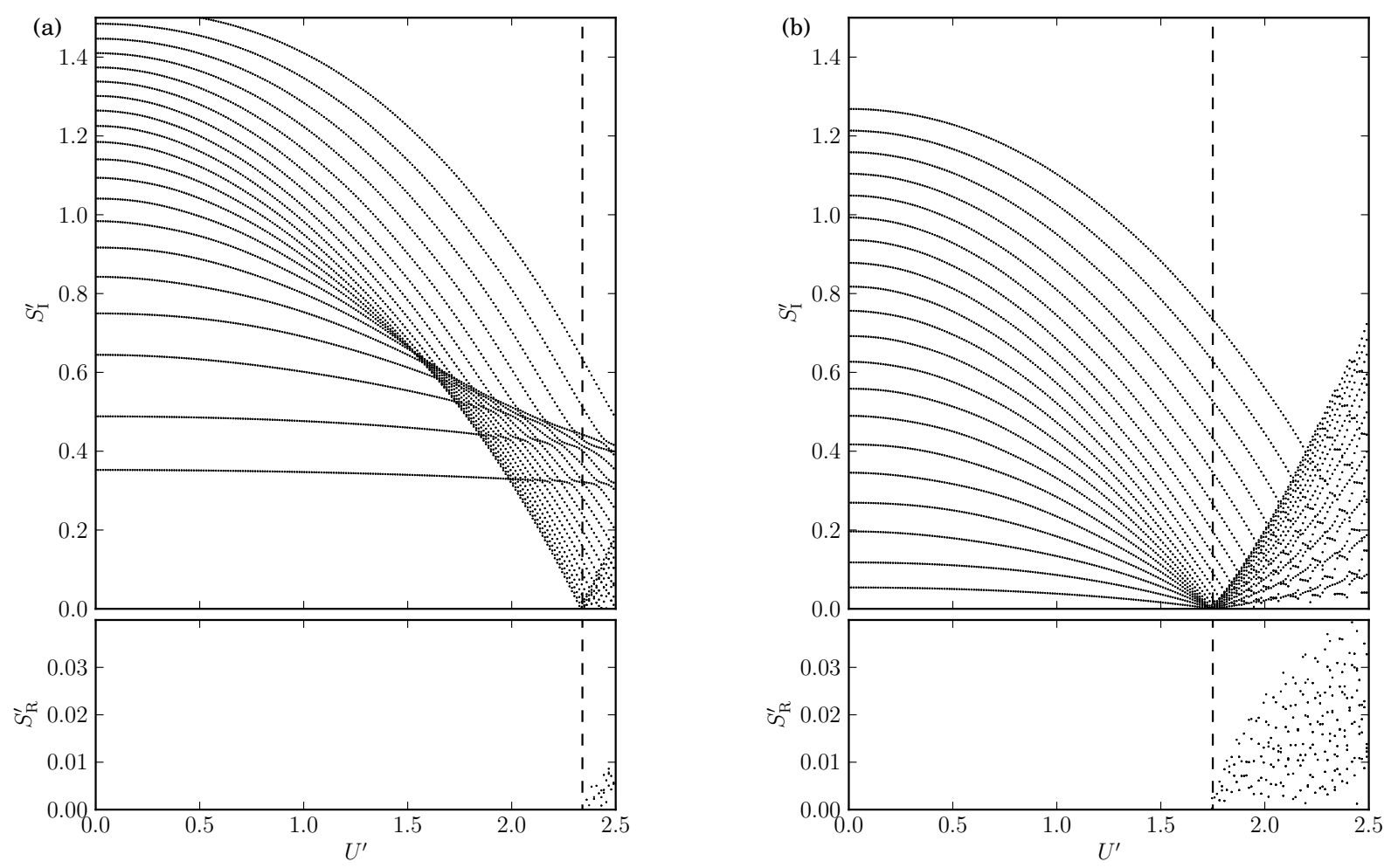

Figure 6: The variation of the real and imaginary parts of the 40 lowest eigenvalues with flow speed for a spring-backed flexible plate with mass ratio $\mu=70.4$ in (a) open flow, and (b) channel flow with $H^{\prime}=0.7$. The vertical dashed line indicates the critical flow speed at which the eigenvalues first have a positive real value. 
(a)

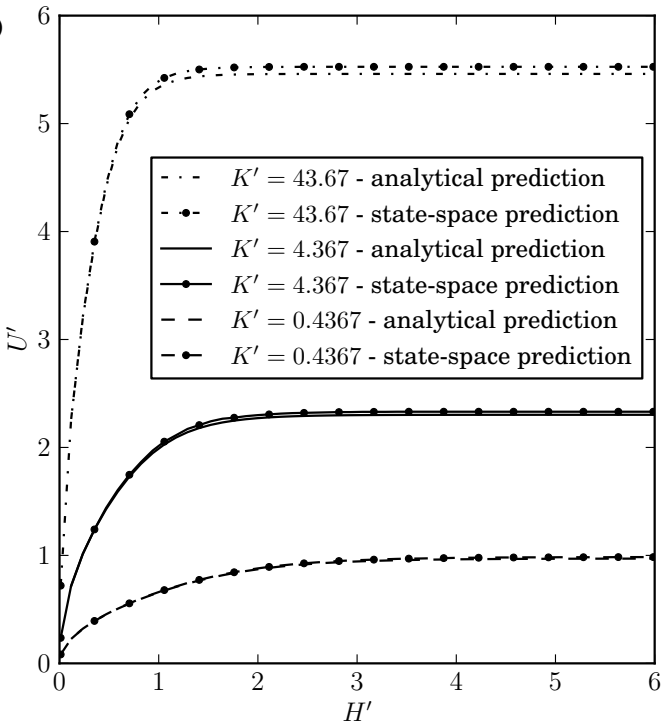

(b)

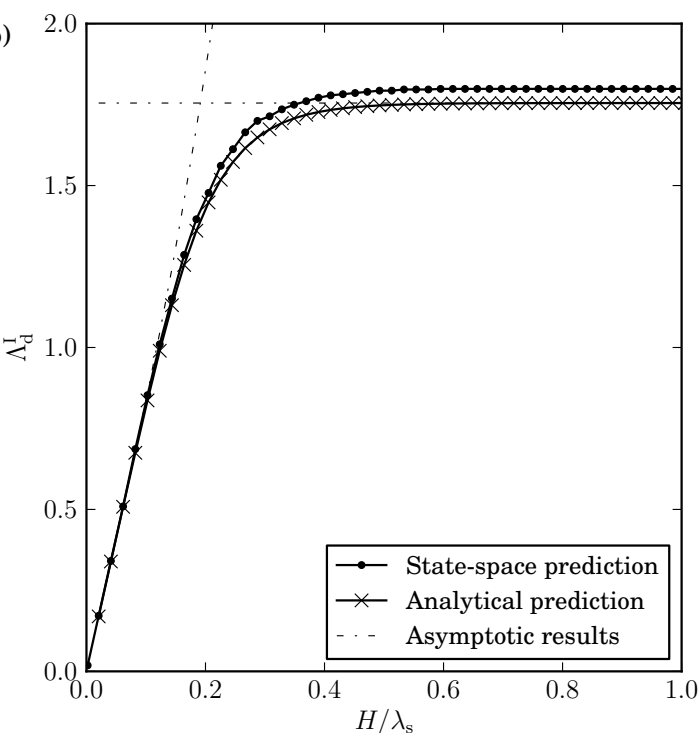

Figure 7: The dependence of divergence-onset flow speed of a spring-backed flexible plate on channel height comparing the results of analytical and state-space forms for three different spring foundations: (a) using the non-dimensional scheme of Lucey and Peake (2003) and (b) a non-dimensional scheme based upon that used in Lucey et al. (1997) including, as dot-dashed lines, the asymptotic results of Eqs. (21b) and (24a) of the analytical predictions. 

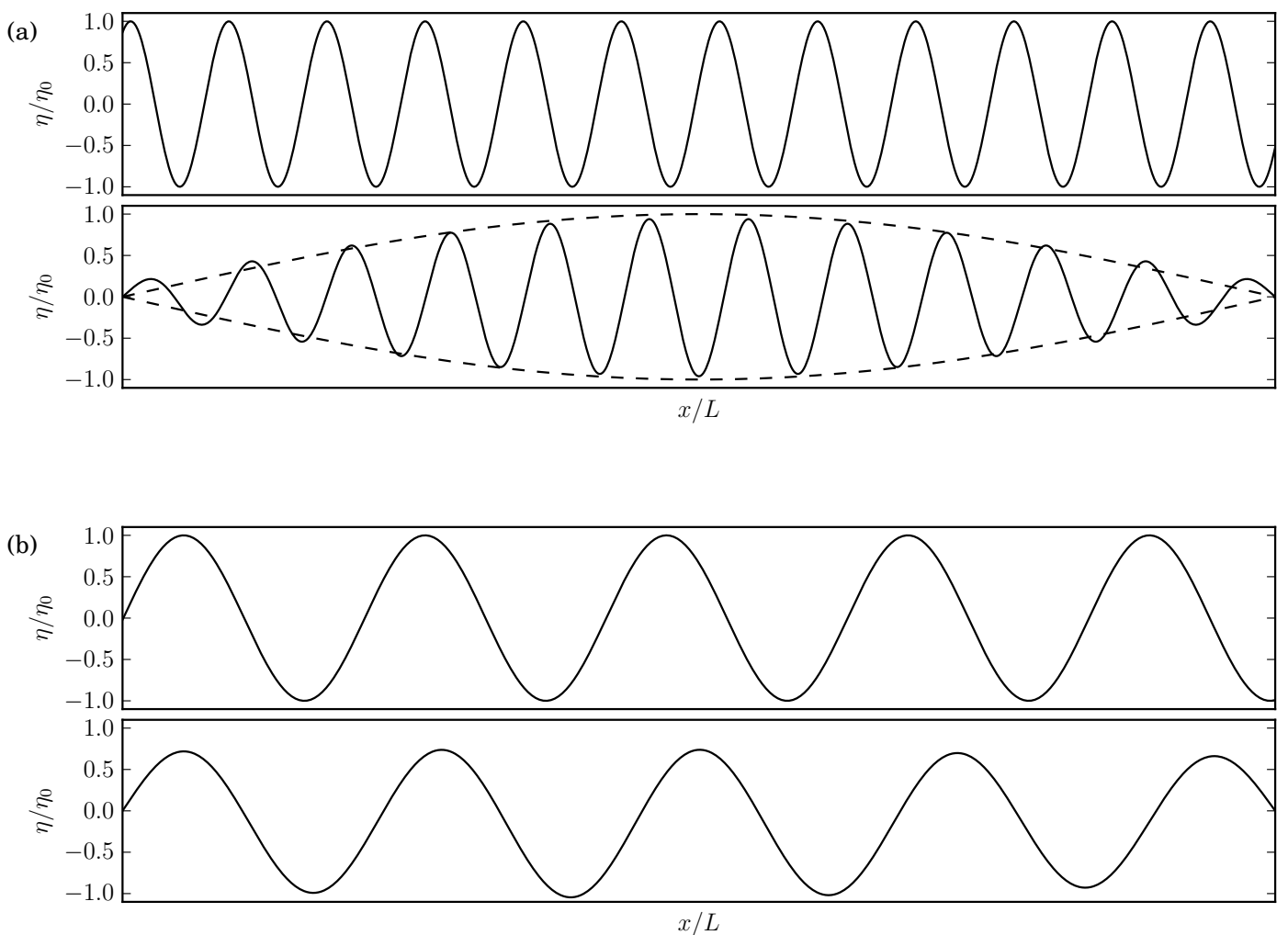

(c)

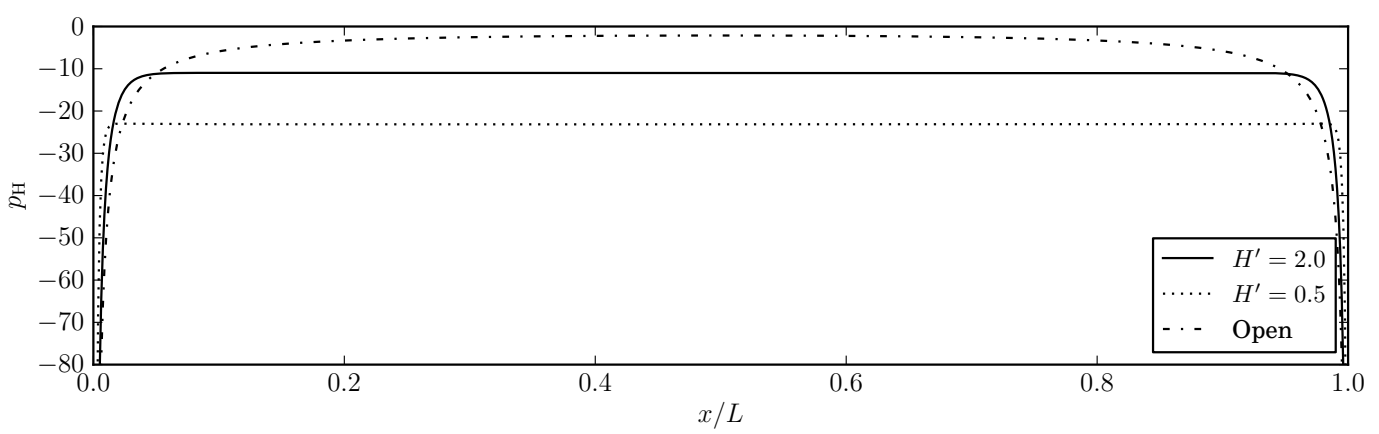

Figure 8: Flexible-wall deformation at divergence onset when $K^{\prime}=4.367$ as assumed in the analytical method (upper) and as predicted by the state-space solution (lower) when: (a) $H^{\prime}=2.0$, and (b) $H^{\prime}=0.5$. (c) The spatial variation of the hydrodynamic-stiffness coefficient for uniform deflection amplitude at divergence onset for the open flow and the two channel heights used for (a) and (b). 